\title{
Correction to: Innovation in Information Systems and Technologies to Support Learning Research
}

Mohammed Serrhini, Carla Silva, and Sultan Aljahdali

\section{Correction to:}

M. Serrhini et al. (Eds.): Innovation in Information Systems

and Technologies to Support Learning Research, LAIS 7, https://doi.org/10.1007/978-3-030-36778-7

In the original version of this book, the affiliation of the volume editor "Carla Silva" was incorrect. This has now been corrected as follows:

Department of Computer Science, School of Management Sciences, Engineering and Technology, Atlantica University, Lisbon, Portugal. 